\title{
Unraveling Molecular Interactions in Liquid-Liquid Phase- Separation of Disordered Proteins by Atomistic Simulations
}

Matteo Paloni, Rémy Bailly, Luca Ciandrini, and Alessandro Barducci*

Centre de Biochimie Structurale (CBS), INSERM, CNRS, Université de Montpellier, 34090 Montpellier, France.

Supporting Information 


\section{Supporting Data}

\section{Sequence of the 236-residue-long NDDX4 used in the MD simulations}

MGDEDWEAEI NPHMSSYVPI FEKDRYSGEN GDNFNRTPAS SSEMDDGPSR RDHFMKSGFA 60 SGRNFGNRDA GECNKRDNTS TMGGFGVGKS FGNRGFSNSR FEDGDSSGFW RESSNDCEDN 120 PTRNRGFSKR GGYRDGNNSE ASGPYRRGGR GSFRGCRGGF GLGSPNNDLD PDECMQRTGG 180 LFGSRRPVLS GTGNGDTSQS RSGSGSERGG YKGLNEEVIT GSGKNSWKSE AEGGES 236

\section{Supporting Table and Figures}

Table S1. Partial charges used for the simulations of isolated phenylalanines.

\begin{tabular}{|l|l|l|}
\hline Atom name & Atom type & Partial charge \\
\hline $\mathrm{N}$ & $\mathrm{N} 3$ & 0.17260 \\
\hline $\mathrm{H} 1$ & $\mathrm{H}$ & 0.19100 \\
\hline H2 & H & 0.19100 \\
\hline H3 & H & 0.19100 \\
\hline CA & CT & -0.00350 \\
\hline HA & H1 & 0.09670 \\
\hline CB & CT & -0.03540 \\
\hline HB1 & HC & 0.02840 \\
\hline HB2 & HC & 0.02840 \\
\hline CG & CA & 0.01070 \\
\hline CD1 & CA & -0.12670 \\
\hline HD1 & HA & 0.13190 \\
\hline CE1 & CA & -0.17150 \\
\hline HE1 & HA & 0.14190 \\
\hline CZ & CA & -0.10830 \\
\hline HZ & HA & 0.12860 \\
\hline CE2 & CA & -0.17150 \\
\hline HE2 & HA & 0.14190 \\
\hline CD2 & CA & -0.12670 \\
\hline HD2 & HA & 0.13190 \\
\hline C & C & 0.76490 \\
\hline OC1 & O2 & -0.80370 \\
\hline OC2 & O2 & -0.80370 \\
\hline & & \\
\hline & & \\
\hline
\end{tabular}




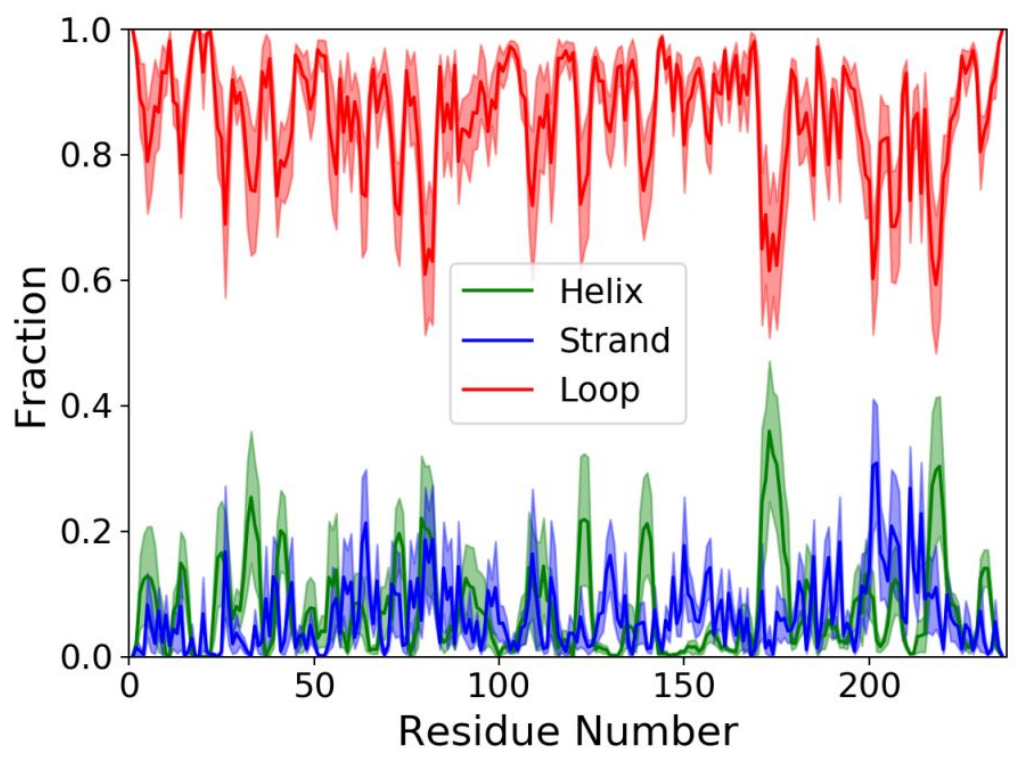

Figure S1. Average secondary structure propensity from MD simulations of NDDX4, shaded areas indicate standard error of the mean $(\mathrm{n}=10)$. 

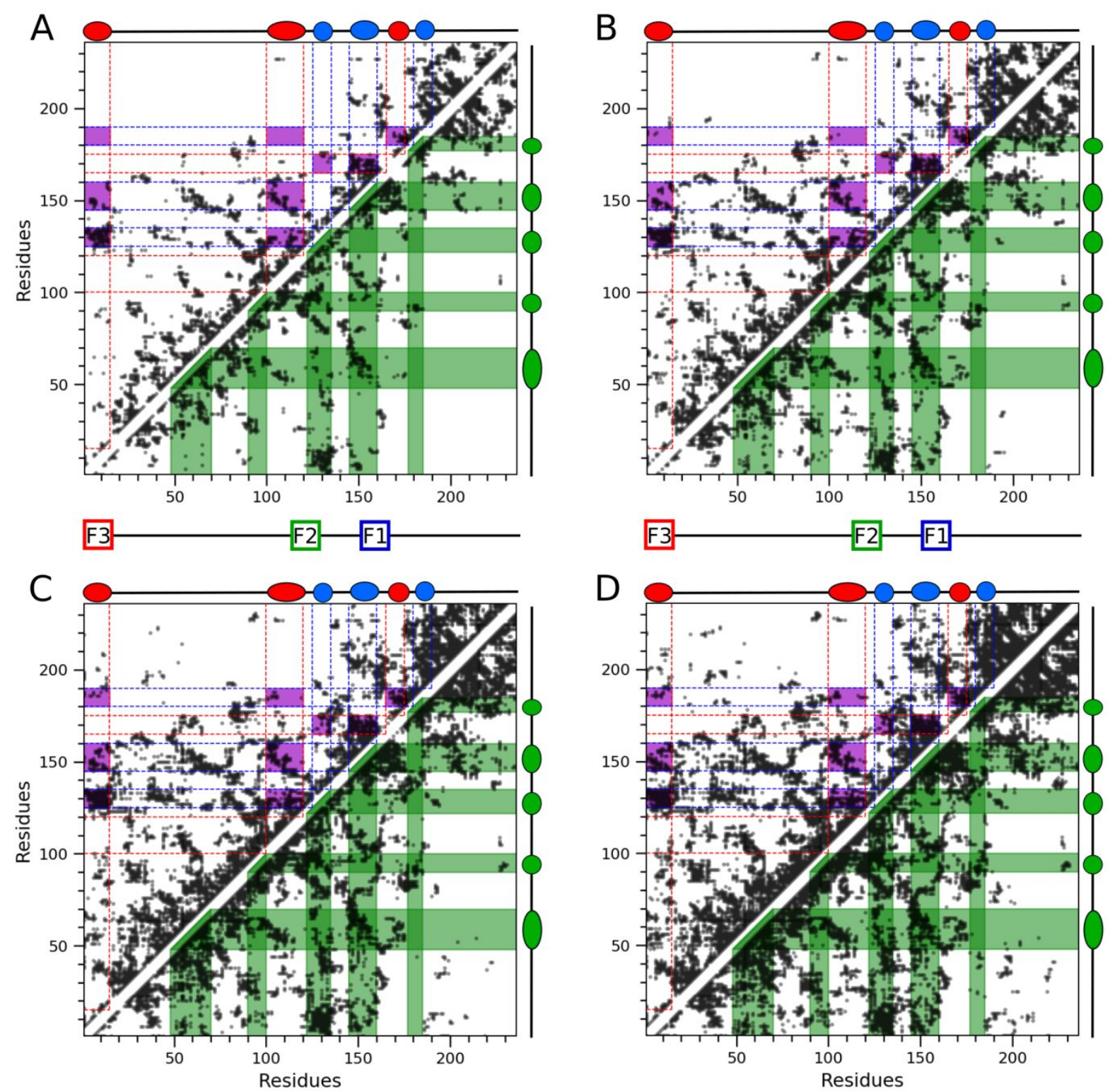

Figure S2. Residue-residue contact probability map as in Fig.1C. Scatter plots represent, respectively, the $10 \%$ (upper left), 15\% (upper right), 20\% (lower left), and 25\% (lower right) most frequent contacts observed in the MD simulations. Position of the fragments used in the simulations of peptides extracted from the NDDX4 sequence is indicated along the $\mathrm{x}$-axis. 


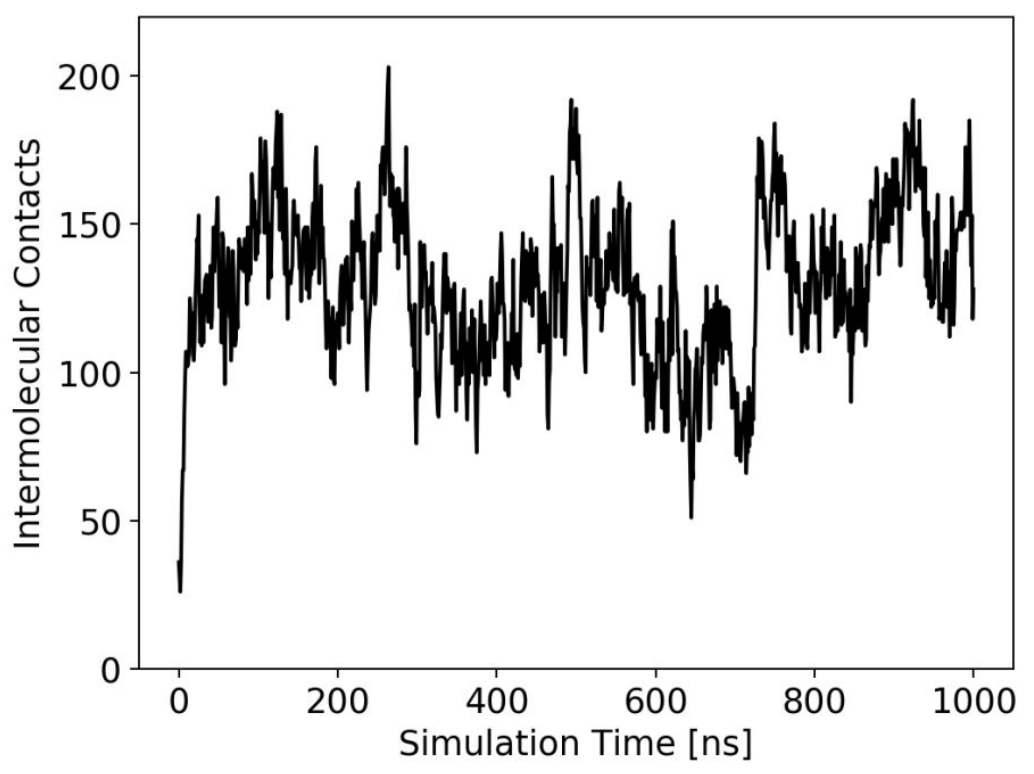

Fig. S3. Number of intermolecular contacts as a function of simulation time.
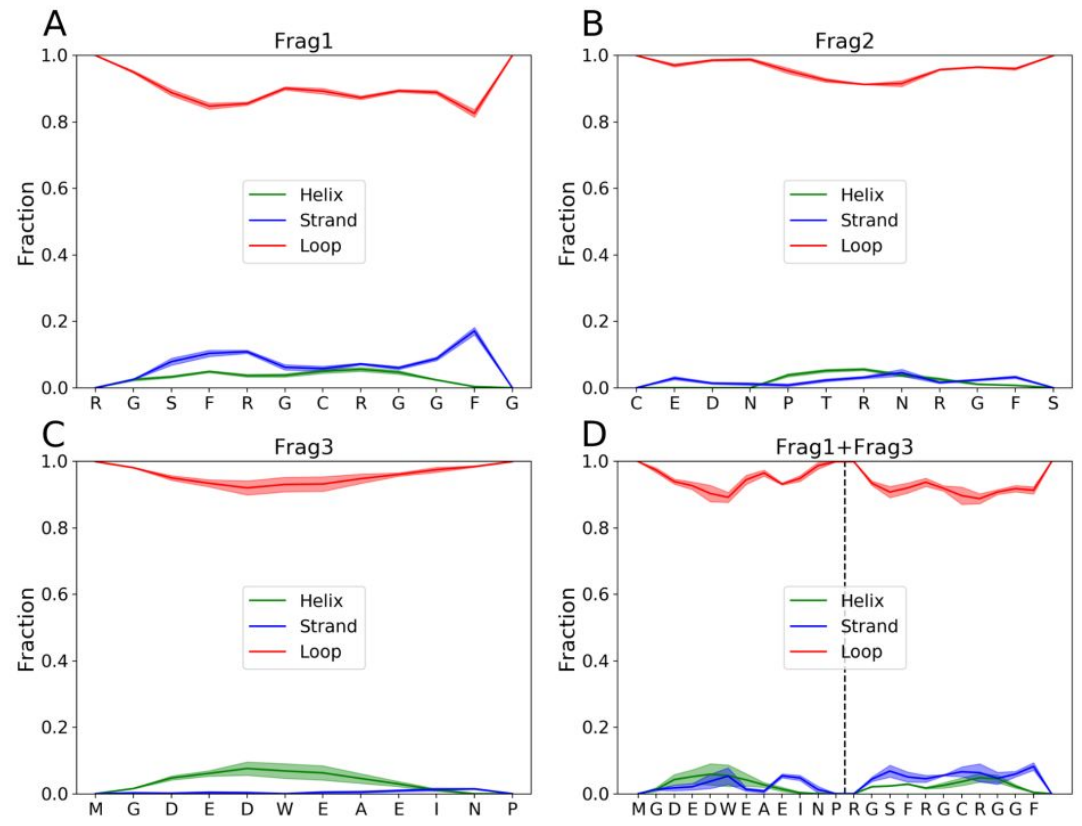

Figure S4. (A-D) Average secondary structure propensity from MD simulations of the simulated fragments. Shaded areas indicate standard error of the mean ( $\mathrm{n}=3$ for all systems except Frag1, for which $\mathrm{n}=5$ ). 


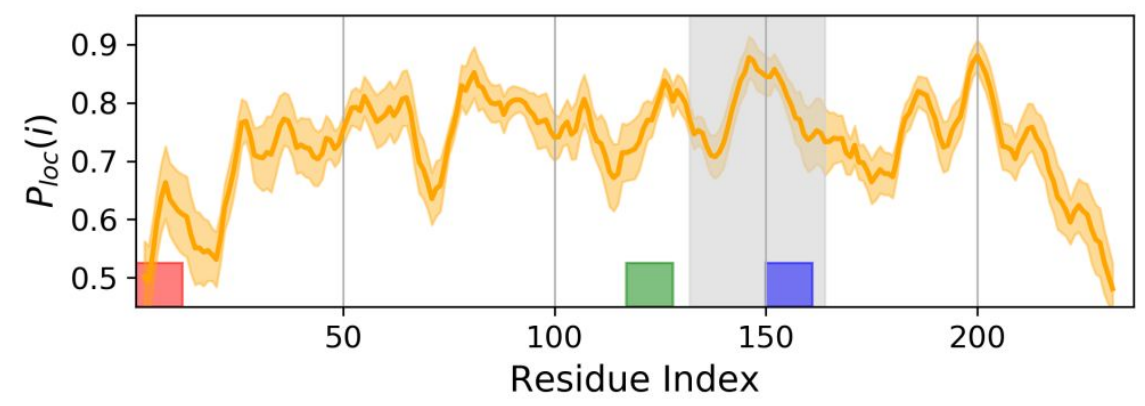

Figure S5. Locally-averaged probability of formation of intramolecular contacts $\mathrm{P}_{\text {loc }}(\mathrm{i})$. Shaded areas represent standard error of the mean $(n=10)$. The grey-shaded area highlights region 132-164, which is necessary for phase separation. Colored squares indicate the position of the 12-residue-long peptides selected to study intermolecular interactions.

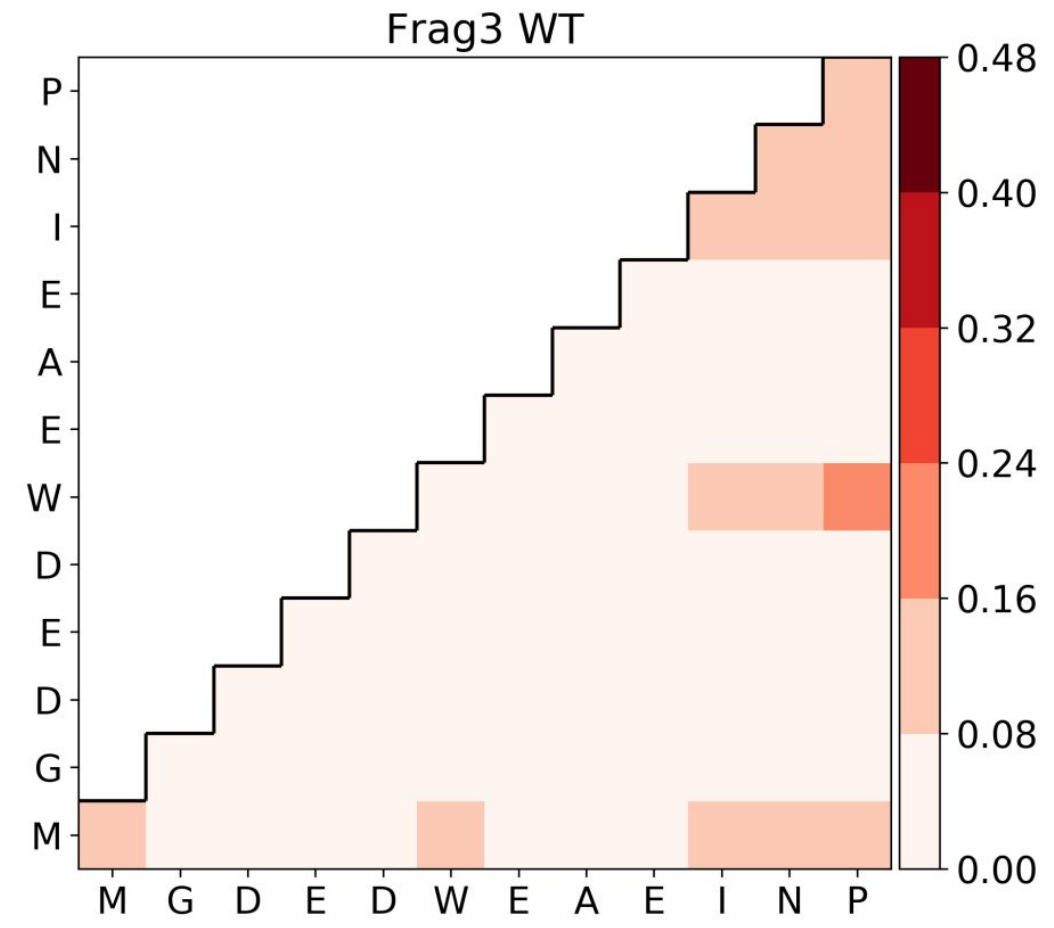

Figure S6. Average intermolecular contact probability from Frag3 MD simulations. 


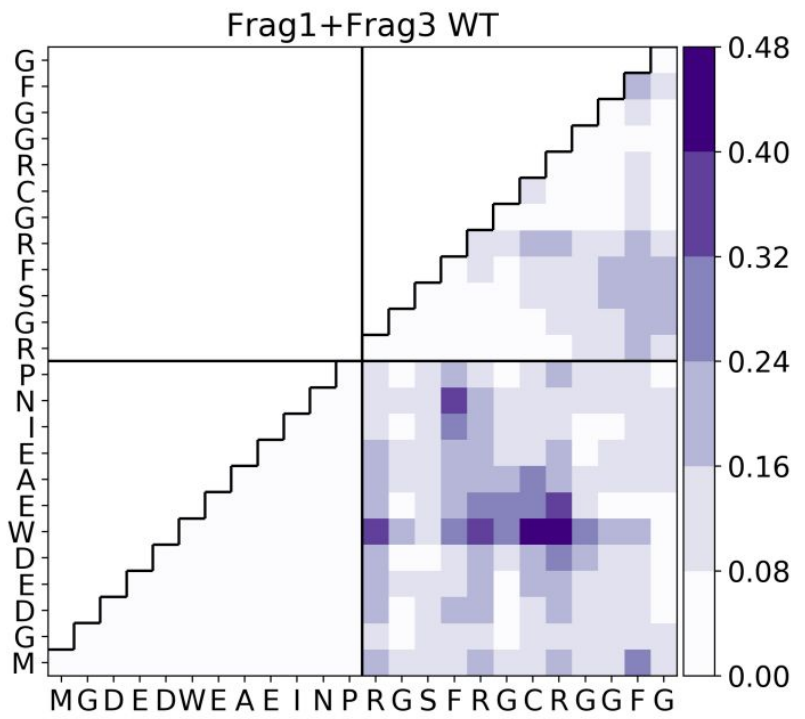

Fig. S7. Average intermolecular contact probabilities of the Frag1+Frag3 WT mixture.

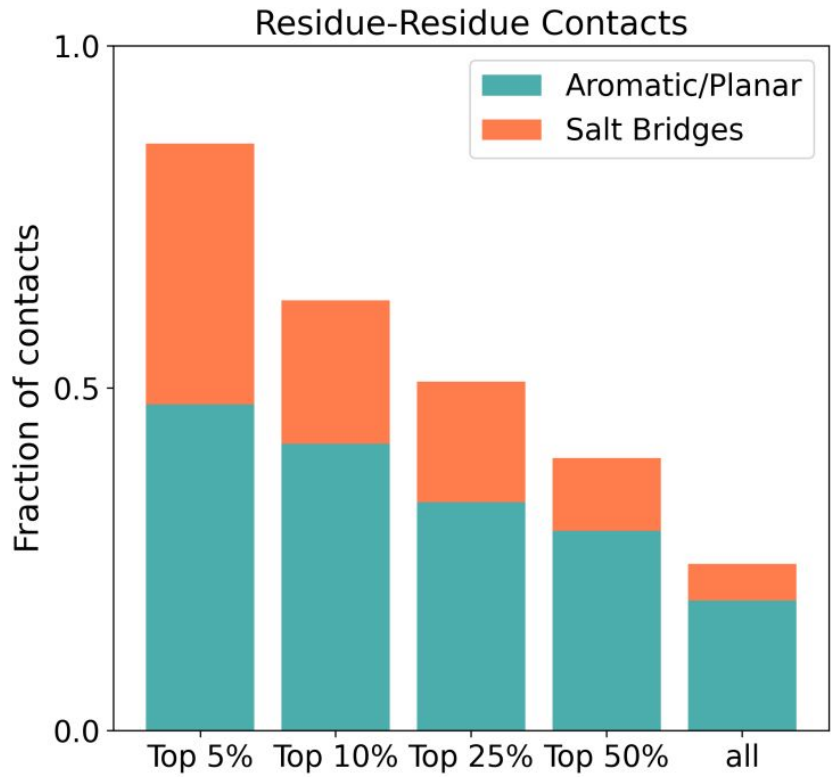

Fig. S8. Fraction of Planar/Aromatic contacts and Salt bridges for different percentiles of the intermolecular contact probability distribution combining Frag1, Frag2, and the heterotypic interaction portion of Frag1+Frag3 simulations. Planar/Aromatic contacts are defined here as contacts between Phe, Trp, Asn, and Arg residues. Salt bridges are defined as contacts between Arg and the negatively-charged Asp and Glu. 

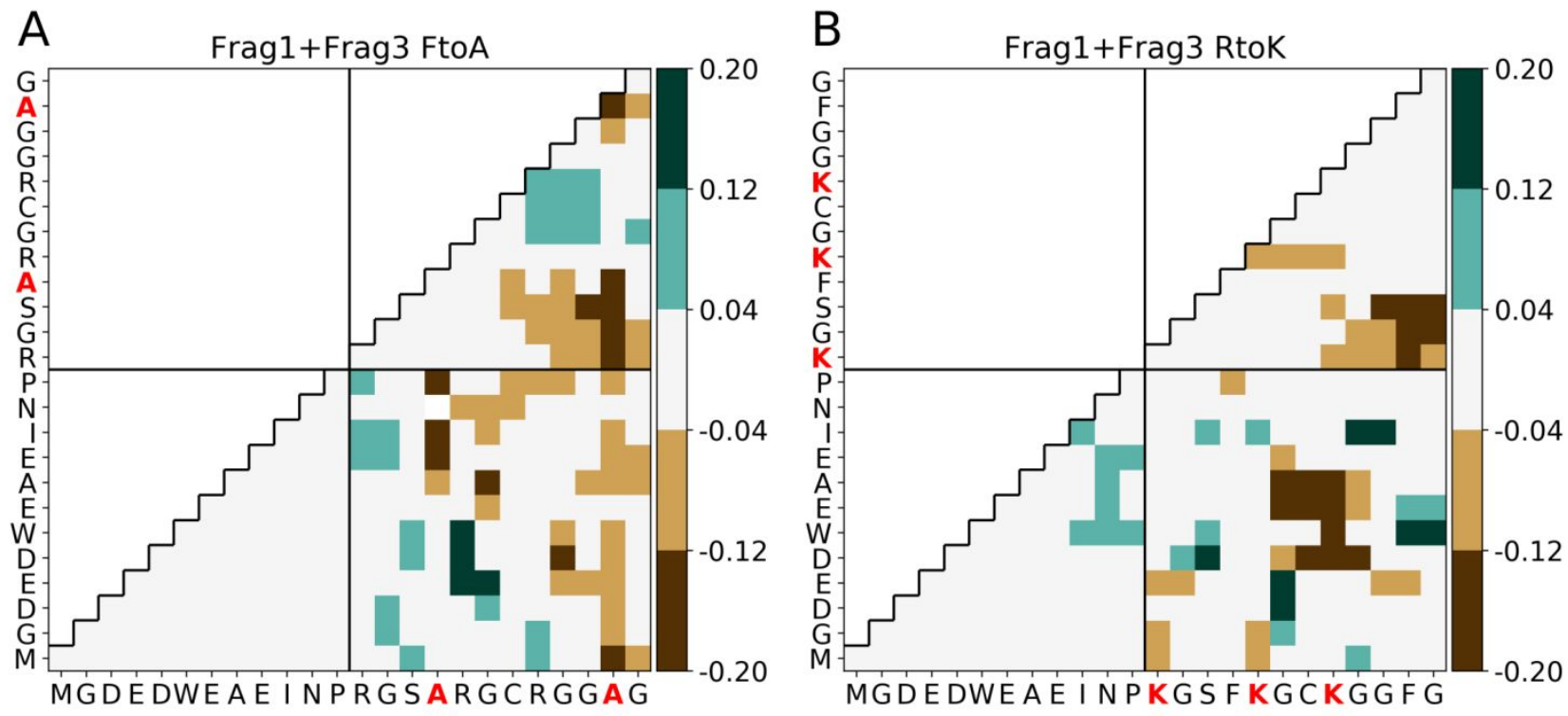

Fig. S9. Variation of the average intermolecular contact probabilities of FtoA (A) and RtoK (B) mutants with respect to WT of Frag1-Frag3 mixture.

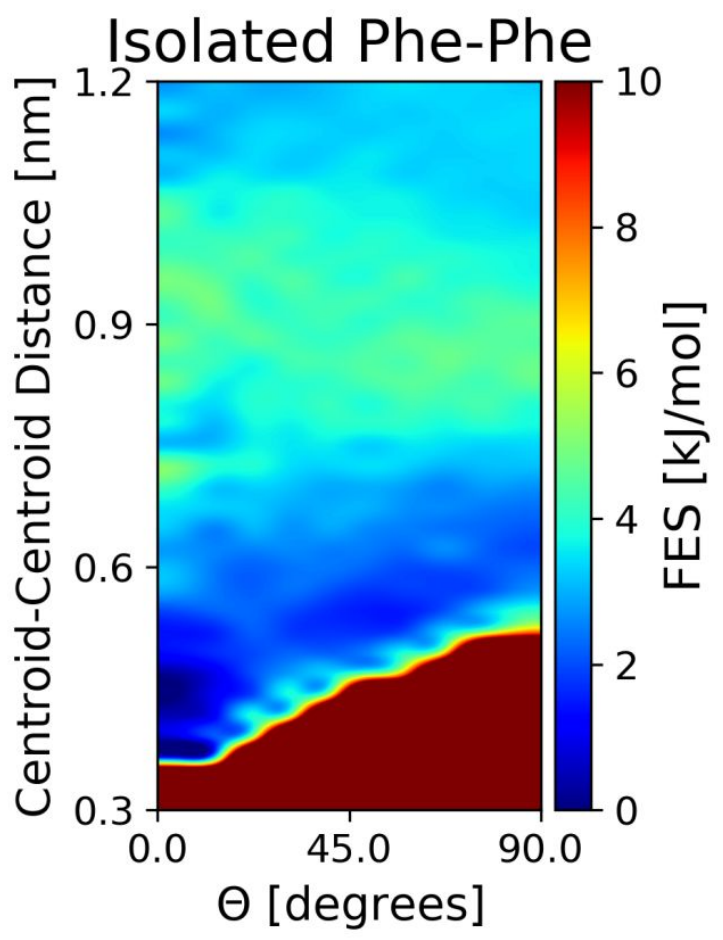

Figure S10. Free energy surfaces for isolated Phe-Phe interactions as a function of the distances between the centroids of guanidinium groups, and their relative orientation $\square$. 

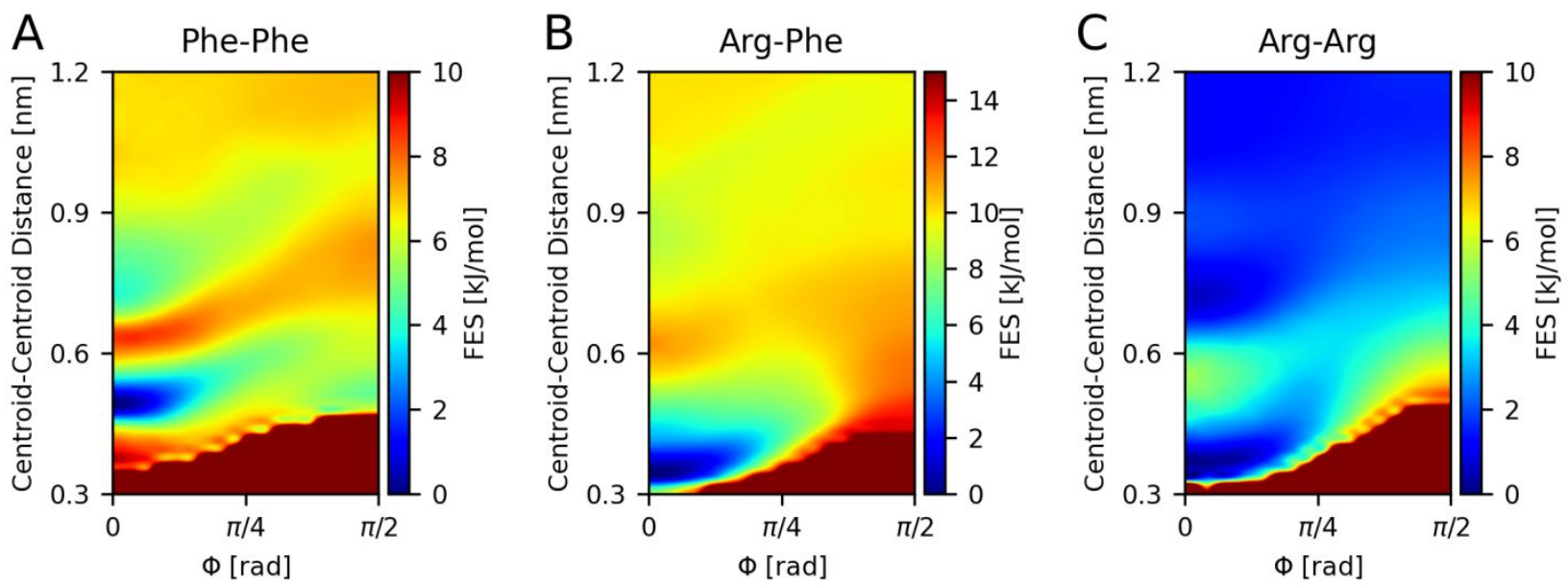

Figure S11. Free energy surfaces for (A) Phe-Phe, (B) Arg-Phe, and (C) Arg-Arg interactions as a function of the distances between the centroids of the planar groups, and their mutual position $\phi$.

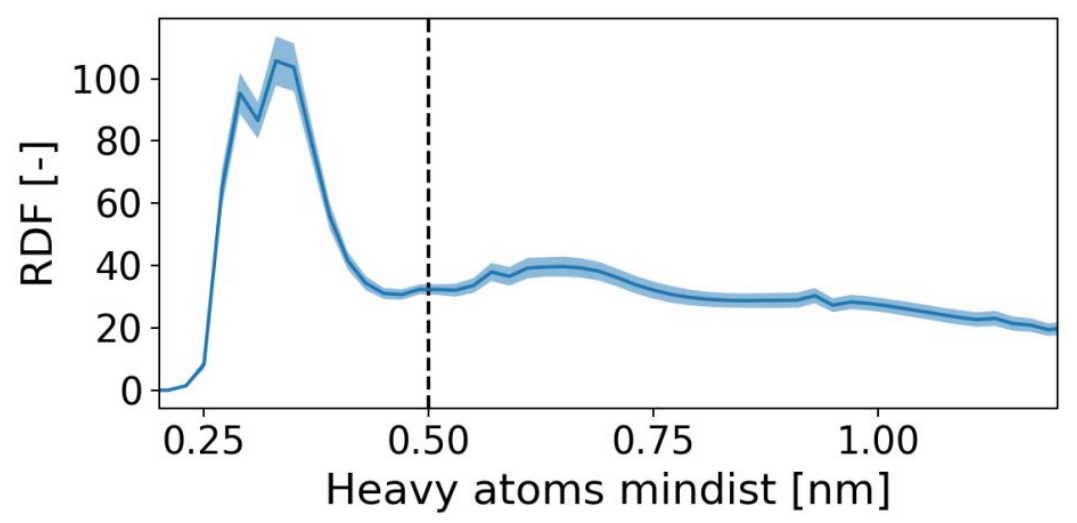

Figure S12. Distribution of minimum inter-residue distances in NDDX4 simulations. Minimum inter-residue distance is defined as the smallest distance between all the heavy atoms of two residues, the distribution was accumulated over all the replicas and normalized as a radial distribution function. Shaded area represents standard deviation. The cut-off used for the definition of the contacts $(0.5 \mathrm{~nm})$ is indicated by the dashed line. 

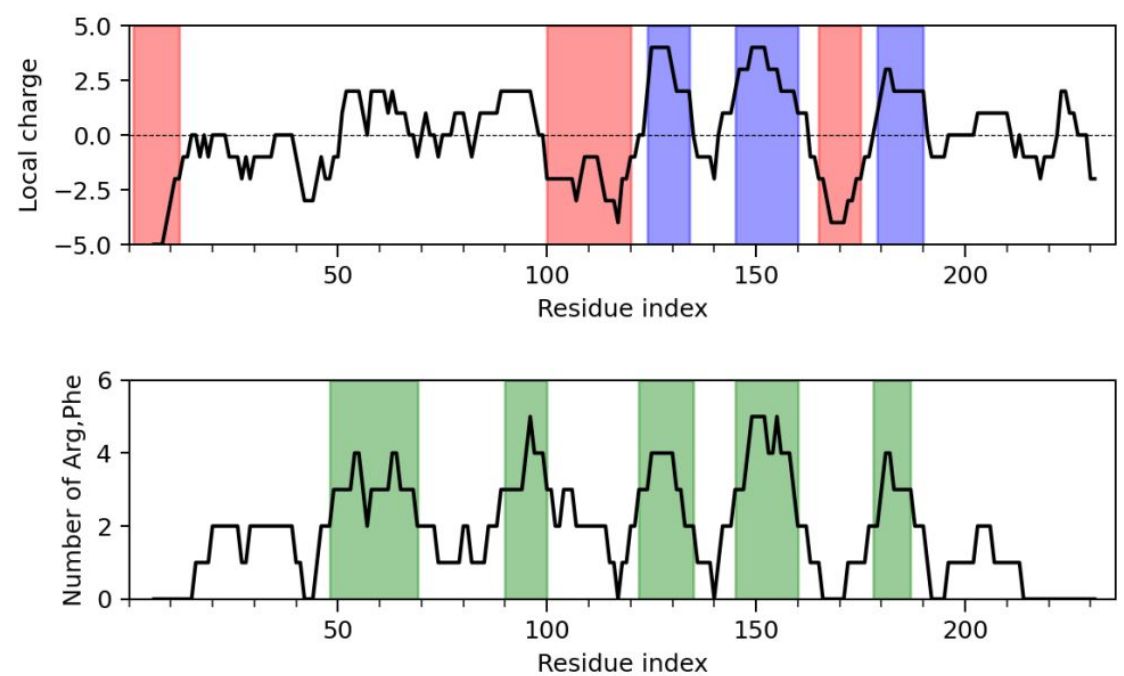

Figure S13. (A) Local charge along the NDDX4 sequence using a moving window of 11 residues. Blue and red shaded areas represent respectively the position of the positively and negatively charged blocks used in Fig. 1C and Fig.S2. (B) Number of arginine and phenylalanine residues along the sequence of NDDX4 using a moving window of 11 residues. Green shaded areas represent the blocks used in Fig.1C and Fig.S2. 